\title{
Séminaires / Seminari 2006
}

\section{Ouverture et gestion d'un cabinet médical}

Contenu (Détails $\rightarrow$ www.fmhservices.ch)

Aménagement - Financement - Administration d'un cabinet médical - Passage du statut de salarié à celui d'indépendant - Assurances - Aspects juridiques et fiscaux - News de l'AMG/SVMED Laboratoire - Atelier questions.

\section{Sponsors}

Assurance des Médecins Suisses, Caisse-Maladie des Médecins Suisses, Medizinische Analytik SA et EMH Editions médicales suisses

\section{Date}

$\begin{array}{llll}\text { No } 28 & \text { Jeudi } & 16 \text { mars } 2006 & \text { CHUV } \\ & \text { Lausanne } & 9.00-17.00 \mathrm{~h} & \\ \text { No } 29 & \text { Jeudi } & 28 \text { septembre } 2006 & \text { HUG } \\ & \text { Genève } & 9.00-17.00 \mathrm{~h} & \end{array}$

\section{Remise/reprise d'un cabinet médical}

Contenu (Détails $\rightarrow$ www.fmhservices.ch) Aspects juridiques - Droit matrimonial et successoral - Mesures architecturales - Assurances - Estimation d'inventaire et goodwill d'un cabinet Conséquences fiscales d'une remise/reprise News de l'AMG/SVMED - Laboratoire - Atelier questions.

\section{Sponsors}

Assurance des Médecins Suisses, Caisse-Maladie des Médecins Suisses et EMH Editions médicales suisses

\section{Date}

$\begin{array}{llll}\text { No 30 } & \begin{array}{l}\text { Jeudi } \\ \text { Lausanne }\end{array} & \begin{array}{l}27 \text { avril } 2006 \\ 9.00-16.30 \mathrm{~h}\end{array} & \text { CHUV } \\ \text { No 31 } & \begin{array}{l}\text { Jeudi } \\ \text { Genève }\end{array} & \begin{array}{l}16 \text { novembre 2006 } \\ 9.00-16.30 \mathrm{~h}\end{array} & \text { HUG } \\ & & & \end{array}$

\section{Radiologie au cabinet médical}

\section{Contenu (Détails $\rightarrow$ www.fmhservices.ch)}

Passage de la radiologie conventionnelle à la radiologie numérique - rentabilité de la radiologie au cabinet médical - nouvelles prescriptions à partir du $1^{\text {er }}$ janvier 2008 - évaluation et acquisition d'installations neuves ou déjà utilisées aperçu du marché et de ses composantes en matière de prix et de prestations.

\section{Frais}

Fr. 300.- (y c. documentation de cours et repas).

\section{Date}

$\begin{array}{llll}\text { No } 32 & \text { Jeudi } & 23 \text { mars 2006 } & \begin{array}{l}\text { Centre de } \\ \text { Ecublens }\end{array} \\ & 9.30-16.00 \mathrm{~h} & \begin{array}{l}\text { formation } \\ \text { Galexis SA }\end{array}\end{array}$

\section{Contabilità e fiscalità (Ticino)}

\section{Contenuto}

L'obiettivo di questo seminario è di dare al medico le conoscenze necessarie per utilizzare autonomamente lo strumento contabile e per operare con più efficienza ed economicità, nonché far comprendere al medico il significato della pianificazione fiscale.

Mediante esempi pratici elaborati in gruppo, verrà illustrato in maniera semplice ma completa il sistema di gestione della contabilità di uno studio medico con effetto anche sulla pianificazione fiscale.

\section{Date}

$\begin{array}{llll}\text { No } 33 & \text { Giovedì } & 18 \text { maggio } 2006 & \text { FMH } \\ & \text { Chiasso } & \text { dalle } 9.00 \text { alle } 16.00 & \begin{array}{l}\text { Services } \\ \text { Fiduciaria }\end{array}\end{array}$

\section{Inscription et information}

Internet www.fmhservices.ch ou FMH Consulting Services, Simone Köpfli, Burghöhe 1, 6208 Oberkirch, Tél. 04192500 77, Fax 041 9210586.

\section{Remarque}

Les adresses des participants aux séminaires dont les coûts sont couverts en partie ou totalement par des sponsors sont communiquées aux sponsors concernés.

\section{Conditions d'annulation}

Pour les séminaires gratuits, un montant est perçu pour absence ou annulation. Il est de:

- Fr. 50.- par personne dans les 15 jours avant;

- Fr. 80.- par personne dans les 7 jours avant le début du séminaire.

Pour les séminaires payants, un montant est perçu pour une absence ou une annulation. Il est de

- $30 \%$ des coûts du séminaire dans les 15 jours avant le début du séminaire;

- 100 \% des coûts du séminaire dans le 7 jours avant le début du séminaire. 